\section{Segmental Box Girder: Deflection Probability and Bayesian Updating}

By Zdeněk P. Bažant, ${ }^{1}$ Fellow, ASCE, and Joong-Koo Kim²

AbStRact: Probabilistic prediction of the confidence limits on long-time deflections and internal forces of prestressed concrete segmental box-girder bridges is tions and internal forces of prestressed concrete segmental box-girder bridges is covelop. The un concrete creep (the prior) is very large, but it can be greatly reduced by Bayesian updating on the basis of short-time measurements of the deflections during construction or of short-time creep and shrinkage strains of specimens made from the same concrete as the bridge. The updated (posterior) probabilities can be obtained by latin hypercube sampling, which reduces the problem to a series of deterministic creep structural analyses for randomly generated samples of random parameters of the creep and shrinkage prediction model. The method does not require linearization of the problem with regard to the random parameters, and a large number of the random parameters can be taken into account. Application to a typical boxgirder bridge with age differences between its segments and with a change of structural system from statically indeterminate to determinate is illustrated numerically. The results prove that design for extreme, rather than mean, long-time deflections and internal forces is feasible. Adoption of such a design approach would improve long-term serviceability of box-girder bridges.

\section{INTRODUCTION}

Prestressed concrete segmental box-girder bridges are structures that are particularly sensitive to their long-time deformations. Deflections much in excess of their calculated values or severe cracking after a longer service period have often been experienced. The consequences, which include costly repairs and even closing of a bridge before the end of its design lifetime, represent a great economic loss. One important cause of this situation is no doubt the fact that, outside the laboratory, creep and shrinkage of concrete are highly uncertain random phenomena, influenced by many random parameters. Predicting only mean deflections, which is the current practice, is insufficient. The design should be based on predictions of extreme deflections that are exceeded with a certain specified small probability.

The probabilistic approach necessitates a realistic creep prediction model, such as the BP model (Bažant and Panula 1978, 1980, 1982). In probabilistic analysis, it would make little sense to use simple but unrealistic models that have large systematic errors resulting from inadequacy of the mathematical model per se, rather than from randomness of influencing parameters. On the other hand, the usefulness of a sophisticated creep prediction model, such as the BP model, is limited unless its use is combined with a probabilistic approach.

${ }^{1}$ Prof. of Civ. Engrg., Dept. of Civ. Engrg., The Tech. Inst., Northwestern Univ., Evanston, IL 60208 .

${ }^{2}$ Grad. Res. Asst., Dept. of Civ. Engrg., The Tech. Inst., Northwestern Univ., Evanston, IL.

Note. Discussion open until March 1, 1990. To extend the closing date one month, a written request must be filed with the ASCE Manager of Journals. The manuscrip for this paper was suberion on June 7,1988 . for this paper was subitted for rever This paper is part of the Journal of Structural Engineering, Vol. 115, No. 10 October, 1989. CASCE, ISSN 0733-9445/89/0010-2528/\$1.00 + \$.15 per page. Paper No. 23976
If creep and shrinkage properties of concrete are predicted solely on the basis of the existing models, the uncertainty is very large. For the BP mode (Bažant and Panula 1978, 1980, 1982), errors that are exceeded with a 5\% probability $(2.5 \%$ on the plus side, $2.5 \%$ on the minus side) are about $37 \%$, while for the simpler but less realistic models of ACI Committee 209/II $(1971,1982)$ and CEB-FIP $(1978)$ they are $75 \%$ and $91 \%$, respectively (Bažant and Panula 1982; Bažant 1988; Zebich and Bažant 1981). As shown in Bažant and Panula (1982), this uncertainty can be greatly reduced if some shorttime measurements are made and Bayesian statistical reasoning applied. An analytical method to do that for long-time creep compliance of concrete was developed in Bažant (1983) and Bažant and Chern (1984). This method, however, works only if the creep law is linearized and if there are only few random parameters (two or three). None of these simplifications is adequate for predicting deflections of an entire box girder.

General applicability can be achieved by combining Bayesian updating with a sampling approach, as formulated in Bažant (1985). This reduces the problem to a series of conventional (deterministic) structural creep analyses for various samples of random parameters. A novel sampling method, called latin hypercube sampling (McKay 1980; McKay et al. 1979, 1976), has been adopted. This sampling method, which was originally applied to direct (nonBayesian) probabilistic prediction of structural creep effects (Bažant and Liu 1985), and was later extended to Bayesian extrapolation of shrinkage data (Bažant et al. 1987), is computationally much more efficient than the method of point estimates used previously (Madsen and Bažant 1983).

In the present study, Bažant's (1985) method used for shrinkage in Bažant et al. (1987) will be applied to short-time measurements during construction in order to improve the predictions of long-time deflections and internal forces in a segmental box girder which undergoes a change of its structural system during construction and has a nonuniform age of concrete. This approach has the potential of substantially reducing the uncertainty of long-time predictions and making it possible to adjust the vertical alignment of the segments during construction, thereby minimizing deviations from the desired vertical profile of the bridge.

\section{Linear Creep Analysis of Segmental Box Girder}

Concrete is assumed to behave as an aging linearly viscoelastic material (Bažant 1982a, 1988). It is characterized by the compliance function $J\left(t, t^{\prime}\right)$ representing the strain of concrete at time $t$ caused by a unit uniaxial stress applied at time $t^{\prime} . J\left(t, t^{\prime}\right)$ is defined, according to the BP model (Bažant and Panula 1978, 1980, 1982), as a sum of the asymptotic elastic compliance, the basic creep compliance, and the drying creep compliance. The shrinkage strain, also defined according to the BP model, is assumed to occur homogeneously throughout the cross section, which is a usual simplification in design. The thermal strains are ignored.

In a typical segmental bridge (Fig. 1), the age of concrete is nonuniform. Let time $t$ represent the age of the oldest segment, numbered $k=1$. The compliance function of segment number $k$ is denoted as $J_{k}\left(t, t^{\prime}\right)$. If the properties of concrete are the same as in the segment number 1 , then $J_{k}\left(t, t^{\prime}\right)=$ $J_{1}\left(t-\Delta_{k}, t^{\prime}-\Delta_{k}\right)=$ compliance function of segment number 1 , where $\Delta_{k}$ is the age difference between the segments $k$ and 1 . 
a) गाम
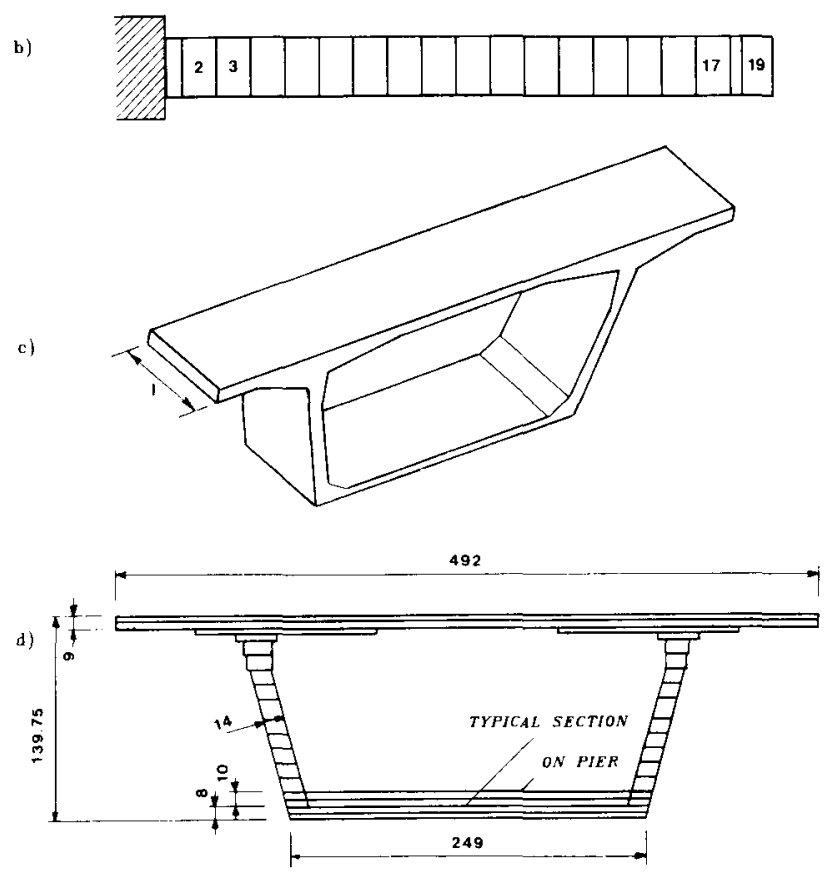

e)

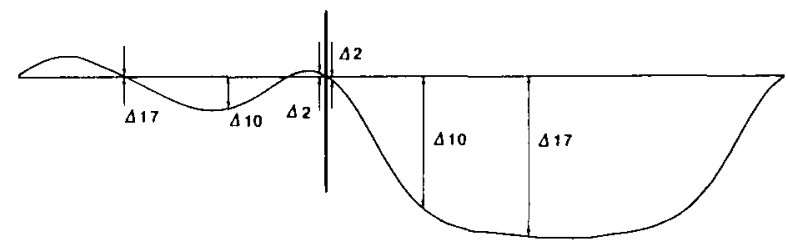

FIG. 1. Bridge Analyzed in Example (Kishwaukee River Bridge, III.): (a) Longitudinal Cross Section; (b) Cantilever (Half-Span) Analyzed; (c) Box Girder Segment; (d) Cross Section Simplified for Analysis; and (e) Elastic Deflection Curve and Deflections Measured (Dimensions in Inches, 1 in. $=25.4 \mathrm{~mm}$ )

For numerical computation, time $t$ is subdivided by discrete times $t_{i}$ ( $i=$ $1,2 \ldots)$ in time steps $\Delta t_{i}=t_{i}-t_{i-1}$. Until time $t_{m}$ the box girder consists of a statically determinate cantilever, and the stress is then constant if the load is constant. We assume that the load due to the own weight of the segment number $k$ is applied instantly at time $t_{k}$ at which this segment is placed in position and prestressed (or freed from the formwork, if cast in situ). Thus, all the stresses vary at $t=t_{k}$ by a sudden jump and subsequently remain constant until time $t_{k+1}$ when the next segment is placed. This results in a staircase history of moment (Fig. 2). At time $t_{m}$ the cantilevers are

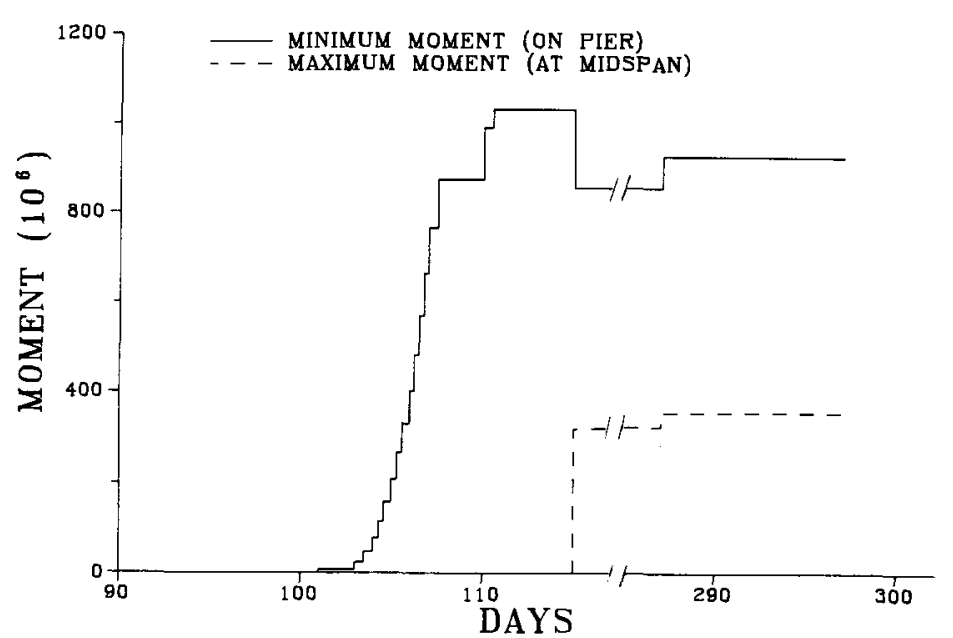

FIG. 2. History of Minimum Bending Moment at Support and Maximum Bending Moment at Midspan Produced by Construction Sequence with Time Steps Considered in Analysis

joined, and afterwards the stresses gradually redistribute due to statical indeterminacy. This causes, after time $t_{m}$, the stresses to vary continuously in time.

For our purposes the box-girder bridge (Fig. 1) may be analyzed as a beam in which the cross sections remain plane and normal. We neglect the shear lag (Křistek and Bažant 1987), for the sake of simplicity. We ignore the effects of nonprestressed steel, and of transverse or vertical prestressing (if any). Perfect bond is assumed for the tendons. For the sake of simplicity, we assume that the two cantilevers joined at midspan are symmetric, and the ages of symmetric segments are the same (Fig. 3). The supports are assumed to prevent rotation but permit free horizontal dilatation of the span.

The cantilevers are made continuous at time $t_{m}$. Since rotations at the cantilever ends are impossible after time $t_{m}$, a redundant bending moment, $X$, gradually builds up at the midspan after time $t_{m}$. Due to symmetry, however, the shear force at midspan is zero, so the structure is only singly redundant (Fig. 3).

The structure is analyzed with a computer program for the layered finite element method. Each segment of the box girder (Fig. 1) represents one finite element, which is subdivided into layers. The reinforcement represents additional elastic layers. The prestressing is implemented by introducing for the prestressed bars initial strains for treatment of creep, and the constitutive law based on the compliance function is converted to the rate-type form corresponding to an aging Maxwell chain model. This is done using the subroutine MATPAR described in Bažant (1982b), and in an improved version by $\mathrm{Ha}$ et al. (1984). The numerical integration in time steps is carried out according to the exponential algorithm for Maxwell chain (Bažant 1982a, 1988).

It may be noted that after the completion of calculations a new creep law 

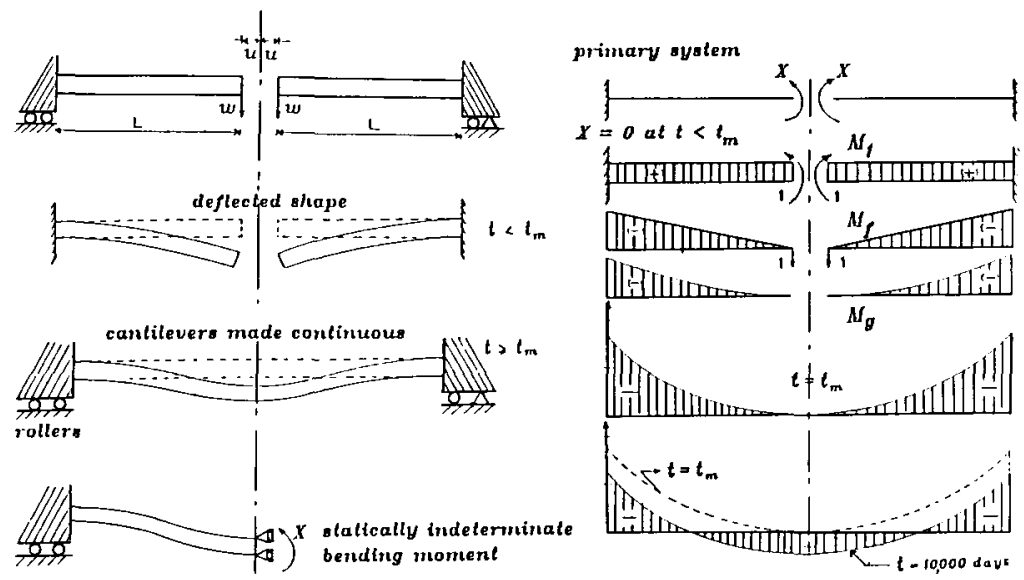

FIG. 3. Change of Structural System and Moment Redistribution

of concrete that is more realistic, simpler to use, and better justified physically was developed (Bažant and Prasannan 1988). Through a certain transformation of time, the model can be reduced to a nonaging Kelvin chain, which would allow a simpler solution method than that used here.

\section{Random Parameters for Creep and Shrinkage}

We adopt the same probabilistic approach as in Bažant and Liu (1985) and Madsen and Bažant (1983); see also Křístek and Bažant (1987) for all the approximations and simplifications involved. For the deterministic basis, the BP model from Bažant and Panula $(1978,1980,1982)$ is used. The random errors of model are introduced through uncertainty factors $\theta_{1}, \theta_{2}, \theta_{3}$ as follows:

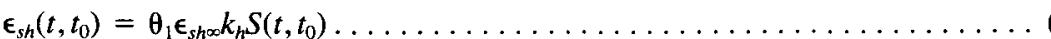

$J\left(t, t^{\prime}\right)=\theta_{2}\left[\frac{1}{E_{0}}+C_{0}\left(t, t^{\prime}\right)\right]+\theta_{3}\left[C_{d}\left(t, t^{\prime}, t_{0}\right)-C_{p}\left(t, t^{\prime}, t_{0}\right)\right] \ldots \ldots \ldots \ldots \ldots$

in which $t_{0}=$ the age at the start of drying; $t^{\prime}=$ the age when the load is applied; coefficients $\epsilon_{\text {sho }}, k_{h}, E_{0}$ and functions $S, C_{0}, C_{d}$ and $C_{p}$ are defined in Bažant and Panula (1978); $E_{0}=$ the asymptotic modulus; $C_{0}\left(t, t^{\prime}\right)=$ the basic creep compliance (i.e., compliance at constant moisture content); $C_{d}\left(t, t^{\prime}, t_{0}\right)=$ an additional compliance due to simultaneous drying; and $C_{p}\left(t, t^{\prime}, t_{0}\right)=$ a further correction due to drying which is negligible except for very thin members after a long period of drying (here we use $C_{p}=0$ ).

In addition to uncertainty factors $\theta_{1}, \theta_{2}$ and $\theta_{3}$, we consider that, in the BP model, the environmental relative humidity $h$, concrete strength $f_{c}^{\prime}$, watercement ratio $w / c$, gravel-cement ratio $g / c$, and cement content $c$ are also random. Thus, we have altogether eight random material parameters $\theta_{i}(i=$ $1,2, \ldots, 8): \theta_{1}, \theta_{2}, \theta_{3}, \theta_{4}=h, \theta_{5}, \theta_{6}=w / c, \theta_{7}=g / c$, and $\theta_{8}=c$.

Because the strength is correlated to the water-cement ratio according to the approximate relation $f_{c}^{\prime}=[1 /(w / c)-0.5] \times 3,300 \mathrm{psi}$, the random parameter for strength has been introduced as the uncertainty factor $\theta_{5}$ of
TABLE 1. Example of Set of 16 Random Samples of Eight Random Parameters for Latin Hypercube Sampling

\begin{tabular}{c|r|r|r|r|r|r|r|r}
\hline $\begin{array}{c}\text { Run } \\
(1)\end{array}$ & $\begin{array}{r}\theta_{1} \\
(2)\end{array}$ & $\begin{array}{r}\theta_{2} \\
(3)\end{array}$ & $\begin{array}{r}\theta_{3} \\
(4)\end{array}$ & $\begin{array}{r}\theta_{4} \\
(5)\end{array}$ & $\begin{array}{r}\theta_{5} \\
(6)\end{array}$ & $\begin{array}{r}\theta_{6} \\
(7)\end{array}$ & $\begin{array}{r}\theta_{7} \\
(8)\end{array}$ & $\begin{array}{r}\theta_{8} \\
(9)\end{array}$ \\
\hline 1 & 13 & 11 & 5 & 10 & 13 & 8 & 16 & 6 \\
2 & 8 & 13 & 4 & 2 & 11 & 4 & 6 & 8 \\
3 & 2 & 9 & 6 & 3 & 2 & 12 & 13 & 2 \\
4 & 5 & 2 & 13 & 16 & 10 & 1 & 4 & 1 \\
5 & 11 & 5 & 15 & 9 & 7 & 10 & 1 & 5 \\
6 & 3 & 4 & 8 & 6 & 4 & 2 & 15 & 7 \\
7 & 7 & 8 & 9 & 11 & 12 & 11 & 10 & 13 \\
8 & 1 & 10 & 11 & 12 & 16 & 5 & 7 & 15 \\
9 & 10 & 14 & 14 & 4 & 15 & 7 & 3 & 4 \\
10 & 4 & 3 & 16 & 13 & 3 & 13 & 2 & 12 \\
11 & 16 & 16 & 10 & 8 & 9 & 3 & 9 & 11 \\
12 & 6 & 12 & 12 & 7 & 1 & 16 & 14 & 9 \\
13 & 15 & 7 & 7 & 14 & 14 & 15 & 8 & 14 \\
14 & 14 & 1 & 3 & 1 & 5 & 14 & 12 & 16 \\
15 & 12 & 6 & 2 & 15 & 8 & 6 & 5 & 3 \\
16 & 9 & 15 & 1 & 5 & 6 & 9 & 11 & 10 \\
\hline \hline
\end{tabular}

this relation; i.e., $f_{c}^{\prime}=\theta_{5}[1 /(w / c)-0.5] \times 3,300$ psi. The expectations and the coefficients of variation of the random parameters are listed in Table 1 ; their values were justified in Madsen and Bažant (1983). The remaining parameters of the BP model are considered, for the present calculation, as deterministic: initial relative humidity $h=1$, temperature $T=23^{\circ} \mathrm{C}$, shape factor $k_{s}=1$, sand-cement ratio $s / c=1.75$, age at the start of drying of each segment $t_{0}=3$ days, and effective thickness of the walls of the box girder $D=12$ in

The calculation was applied, as an example, to the Kishwaukee River Bridge in Illinois, for which the concrete mix per cubic yard was: $658 \mathrm{lb}$ of cement, $289 \mathrm{lb}$ of water, 1,864 lb of gravel, 1,150 lb of sand, and $4.5 \%$ of air. The slump was $3 \pm 1$ in. The wall thickness $D$ is variable within the cross section and along the box girder. Although the effect of this variation is quite important for the differences in the creep properties, it must be left for a separate, more-extensive investigation dealing with the effect of moisture diffusion through the walls of the girder. In practice, often the values of the initial prestressing forces at anchors as well as the friction losses along the tendon are not controlled very accurately. All the values $F_{k}\left(t_{k}\right)$ should then also be considered as additional random variables $\theta_{9}, \theta_{10}, \theta_{11}, \ldots$

It should also be kept in mind that parameters $\theta_{1}, \ldots, \theta_{8}$ actually represent a random field over the box girder. Here we assume that the value of each of them is uniform over the entire girder, which is a simplification. Furthermore, the environmental humidity is in reality a random process in time (Bažant and Wang 1983), as well as a random field along the surface.

\section{Bayesian Statistical Prediction by Sampling}

In the initial work on Bayesian statistical extrapolation of measured shorttime creep compliance (Madsen and Bažant 1983), an analytical solution was 
rendered possible by a simplification and transformation which made the response a linear function of only two random parameters. Such a simplification is insufficient for predicting the response of a structure; the dependence on random parameters in nonlinear and more than just two random material parameters are needed. Bažant (1985) showed that, for nonlinearizable problems, Bayesian statistical predictions can in general be obtained by a certain modification of the latin hypercube sampling (McKay 1980 McKay et al. 1976, 1979). Bažant et al. (1987) applied this method to shrinkage extrapolation. We will apply this method to a bridge.

Let $Y_{i}(i=1,2, \ldots, I)$ at times $t_{i}$ be the long-time effects of creep or shrinkage that we want to predict, and let $X_{m}(m=1,2, \ldots, M)$ at times $t^{\prime}$ be the short-time effects of creep or shrinkage that have been measured and which we want to use for improving the predictions. $Y_{i}$ may be either the long-time creep or shrinkage strains, or the long-time structural effects, such as the maximum deflection of the box girder, the maximum bending momen or the span shortening at, say 5, 10, 20, and 40 years. $X_{m}$ may be, for example, the strains of a control cylinder or the box girder deflections at, say, 5 days, 7 days, 28 days, and 5 months.

The values of $Y_{i}$ and $X_{m}$ that may be predicted on the basis of the available information (i.e., without taking the measurements $X_{m}$ into account) will be denoted as $Y_{i}^{\prime}$ and $X_{m}^{\prime}$. The values of $Y_{i}$ may be predicted as certain known functions $y_{i}$ of the random parameters, i.e., $Y_{i}^{\prime}=y_{i}(\boldsymbol{\theta})$, where $\boldsymbol{\theta}=\left(\theta_{1}, \theta_{2}, \ldots, \theta_{N}\right)$ $=$ vector of random parameters. These functions need not be defined by an explicit formula; they are normally defined by a computer algorithm (program), which is the case for the present bridge. Predictions can be also obtained for the short-time effects, and their predicted values, which generally differ from the measured values $X_{m}^{\prime}$, may again be regarded as functions of $\boldsymbol{\theta} ; X_{m}^{\prime}=x_{m}(\boldsymbol{\theta})$. Functions $y_{i}(\boldsymbol{\theta})$ and $x_{m}(\boldsymbol{\theta})$ are nonlinear. They are defined by the finite element program for box-girder deflections or bending moments.

Statistical analysis of numerous test data from the literature yielded certain, albeit limited, information on the statistical properties of the random parameters $\theta_{1}, \ldots, \theta_{N}$ (Bažant and Panula 1978, 1980, 1982; Madsen and Bažant 1983; Tsubaki et al. 1986; Bažant and Zebich 1983). This may be regarded as the prior statistical information, characterized by the prior probability density distributions $f_{n}^{\prime}\left(\theta_{n}\right)$ with means $\bar{\theta}_{n}^{\prime}$ and standard deviations $s_{n}^{\prime}$ $(n=1,2, \ldots, N)$. With this knowledge it is possible to predict the statistical distributions $f^{X^{\prime}}$ of $X_{m}^{\prime}=x_{m}(\boldsymbol{\theta})$ and compare them to the available short-time measurements $X_{m}$ of known distributions $f_{m}^{X}$. The objective is to use this comparison to improve, or update, the statistical information on the random parameters $\theta_{n}$. This improved statistical information may be characterized by updated, or posterior, distributions $f_{n}^{\prime \prime}\left(\theta_{n}\right)$, from which the updated, or posterior, distributions $f_{i}^{Y^{\prime \prime}}\left(Y_{i}^{\prime \prime}\right)$ of the long-time effects $Y_{i}$ may be obtained according to functions $y_{i}(\boldsymbol{\theta})$. The double primes are used to distinguish the posterior characteristics from the prior characteristics, which are labeled by single primes.

As shown in general by Bažant (1985) and applied to shrinkage by Bažant et al. (1987), the method of latin hypercube sampling can be effectively extended to problems of the present type. In this sampling method, the known distribution $f_{i}^{\prime \prime}\left(\theta_{n}\right)$ of each input parameter $\theta_{n}$ is partitioned into $K$ intervals $\Delta \theta^{(k)}$ (strata) of equal probability $1 / K(k=1, \ldots, K)$. The subdivision may be obtained according to the cumulative probability distribution, as illus-

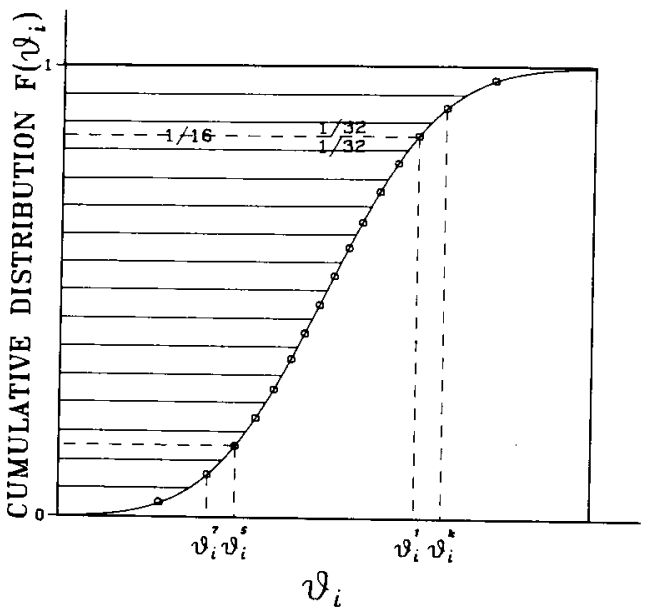

FIG. 4. Subdivision of Random Parameter Range into Intervals of Equal Probability and Determination of Sampling Values

trated in Fig. 4. The number of random parameter samples in latin hypercube sampling must be chosen to be the same as the number of computer runs to be made, and from each interval the random parameter value must be sampled exactly once; i.e., it must be used in one and only one computer run. The sampled value need not be taken randomly from the interval, but may be taken at the centroid of the interval (Fig. 4).

The random selection of the intervals $\Delta \theta^{(k)}$ to be sampled for a particular computer run can be carried out as follows. With each random parameter one may associate a sequence of integers representing a random permutation of the integers $1,2, \ldots, K$. For 16 intervals $(K=16)$, such random permutations are illustrated in the columns of Table 1 . A different random permutation is used for each column (each $\theta_{n}$ ). Thus, each interval number appears in each column of Table 1 once and only once. The individual latin hypercube samples are then represented by the rows in Table 1 . According to the creep or shrinkage prediction model, one can then calculate for each such sample $\theta_{n}^{(k)}(n=1, \ldots, N)$ the effects $X_{m}^{\prime(k)}$ that correspond to the measured short-time effects $X_{m}$, and also the corresponding long-time effects $Y_{i}^{\prime(k)}$. The mean prior prediction $\bar{X}_{m}^{\prime}$ for the measured effects $X_{m}$, the mean prior prediction $Y_{i}^{\prime}$ of the long-time effects, and the corresponding standard deviations then are

$\bar{X}_{m}^{\prime}=\frac{1}{K} \sum_{k=1}^{K} X_{m}^{\prime(k)}, \quad s_{m}^{X^{\prime}}=\left[\frac{1}{K} \sum_{k=1}^{K}\left(X_{m}^{\prime(k)}-\bar{X}_{m}^{\prime}\right)^{2}\right]^{1 / 2}$

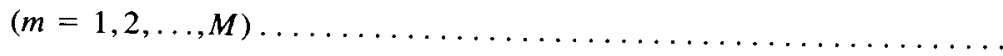

$\bar{Y}_{m}^{\prime}=\frac{1}{K} \sum_{k=1}^{K} Y_{m}^{\prime(k)}, \quad s_{m}^{Y^{\prime}}=\left[\frac{1}{K} \sum_{k=1}^{K}\left(Y_{m}^{\prime(k)}-\bar{Y}_{m}^{\prime}\right)^{2}\right]^{1 / 2}$

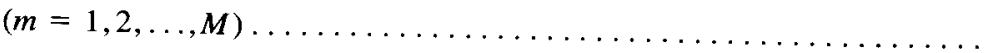



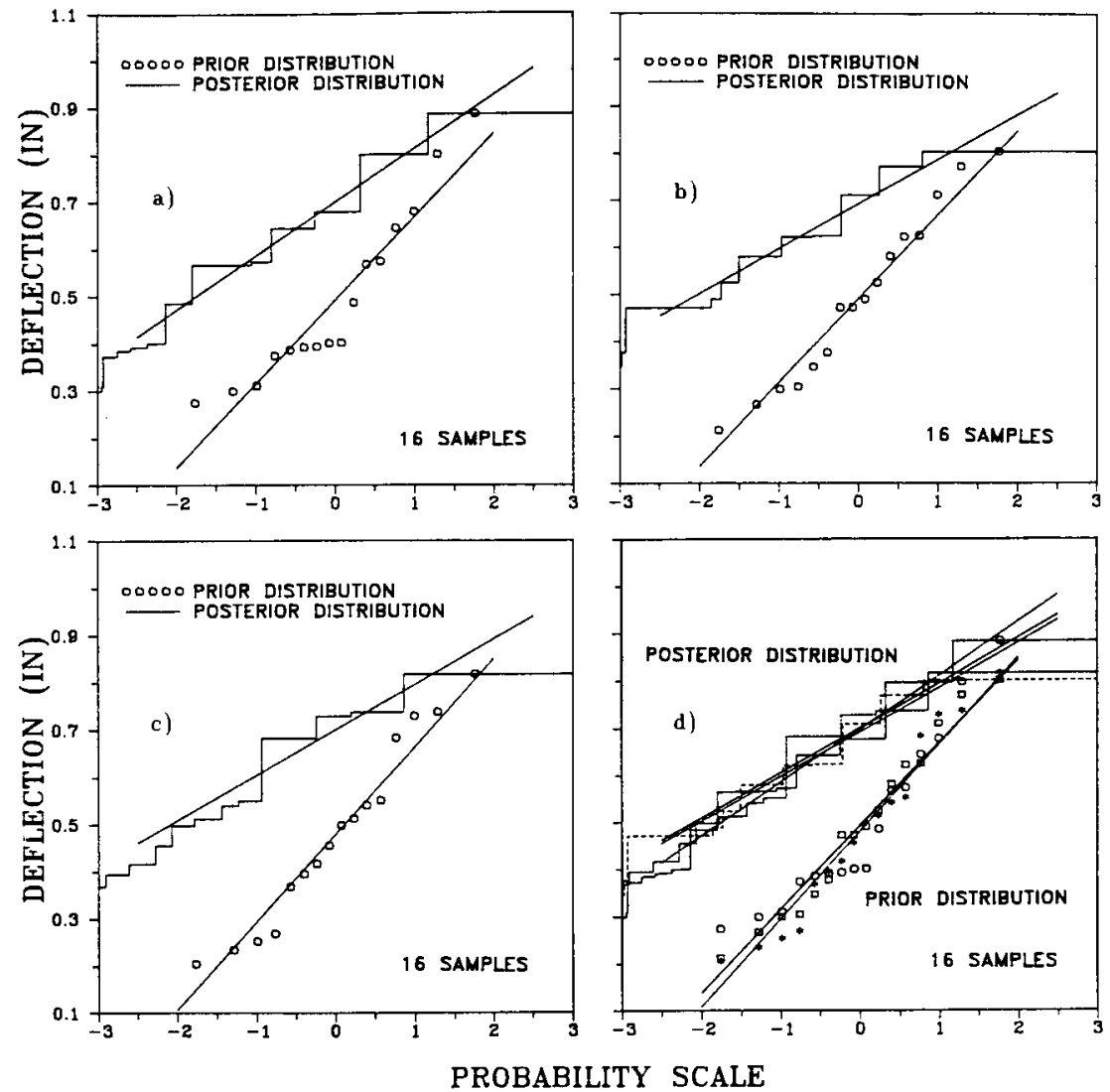

FIG. 5. Typical Cumulative Histograms of Deflection of 17th Segment at 45 yr for Various Sets of 16 Samples with 16 Intervals (in Normal Probability Scale) (1 in. $=25.4 \mathrm{~mm}$ )

Typical cumulative histograms of the prior distributions of $X_{m}$ or $Y_{i}$, as obtained by latin hypercube sampling, are shown in Figs. 5-6. For further plots of such histograms, see Bažant and Liu (1985), where all the predictions are non-Bayesian.

Since one calculated value $Y_{i}^{(k)}$ is obtained for each random sample $\boldsymbol{\theta}^{(k)}=$ $\left(\theta_{i}^{(k)}, \ldots, \theta_{N}^{(k)}\right)$; i.e., $Y_{i}^{(k)}=y_{i}\left(\boldsymbol{\theta}^{(k)}\right)$, the posterior probability of $Y_{i}^{(k)}$ is the same as the posterior probability of $\theta_{i}^{(k)}$; i.e., $P^{\prime \prime}\left(Y_{i}^{(k)}\right)=P^{\prime \prime}\left(\theta_{i}^{(k)}\right)$. While the prior probabilities of all latin hypercube samples $\theta_{i}^{(k)}$ and of the corresponding $X_{m}^{(k)}$ and $Y_{i}^{(k)}$ are equal, the posterior ones are not. The posterior probability is a conditional probability, the condition being that the values $X_{1}, \ldots, X_{M}$ have been observed; i.e., $P^{\prime \prime}\left(X_{m}^{(k)}\right)=P^{\prime \prime}\left(Y_{i}^{(k)}\right)=P^{\prime \prime}\left(\boldsymbol{\theta}^{(k)}\right)=P\left(\boldsymbol{\theta}^{(k)} \mid \mathbf{X}\right)$ where $\mathrm{X}$ $=\left(X_{1}, X_{2}, \ldots, X_{M}\right)=$ vector of the measured creep or shrinkage effects on which the updating is based. According to Bayes theorem (Ang and Tang 1975; Benjamin and Cornell 1970)

$P^{\prime \prime}\left(X_{m}^{(k)}\right)=P^{\prime \prime}\left(Y_{i}^{(k)}\right)=P^{\prime \prime}\left(\boldsymbol{\theta}^{(k)}\right)=P\left(\boldsymbol{\theta}^{(k)} \mid \mathbf{X}\right)=c_{1} L\left(\mathbf{X} \mid \boldsymbol{\theta}^{(k)}\right) P^{\prime}\left(\boldsymbol{\theta}^{(k)}\right)$
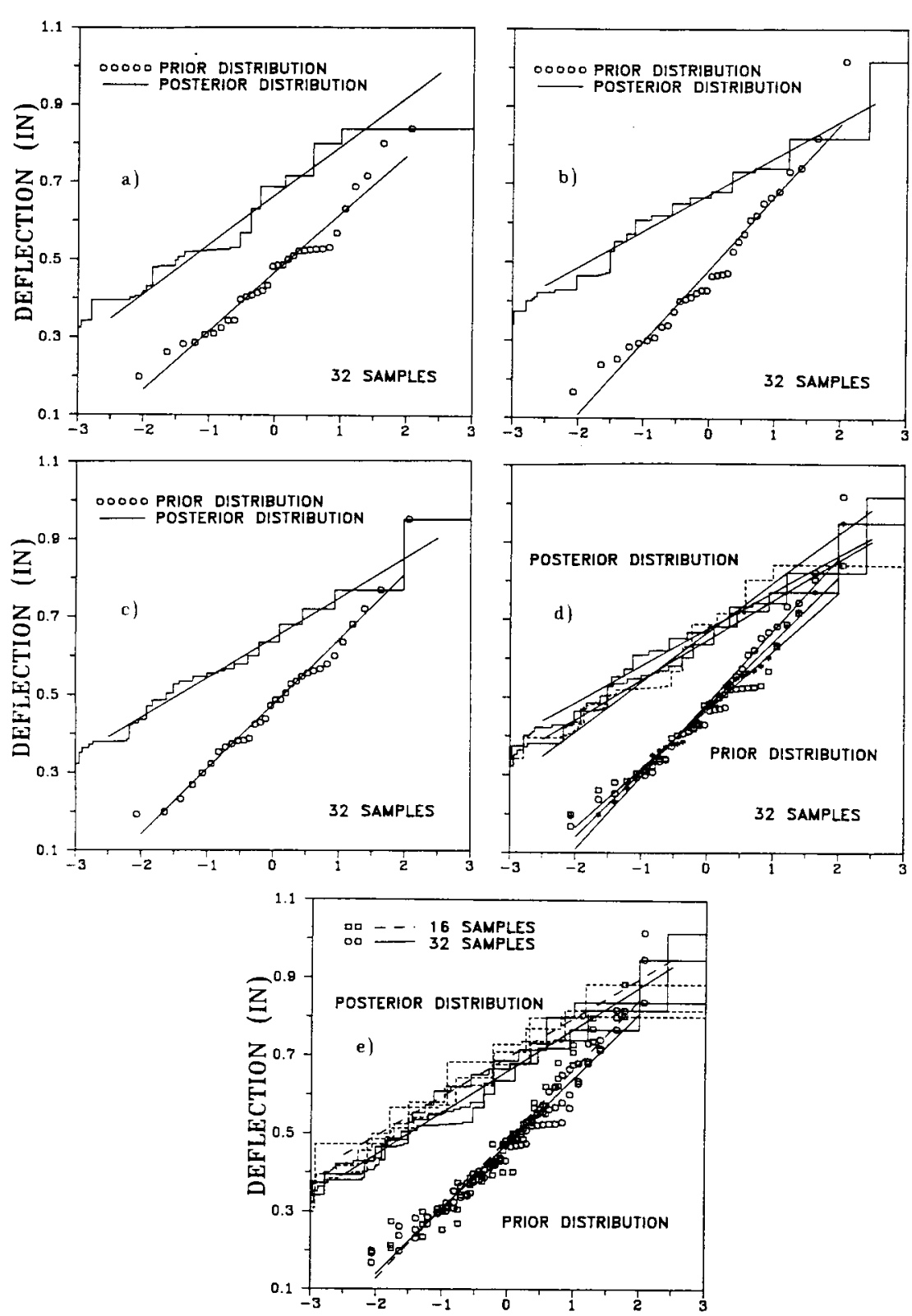

PROBABILITY SCALE

FIG. 6. Histograms: $(a-d)$ Same As Fig. 5 , But for Sets of 32 Samples with 32 Intervals; (e) Comparison of Results for Both 16 and 32 Samples in Each Set 
Here $L\left(\mathbf{X} \mid \boldsymbol{\theta}^{(k)}\right)=L\left(X_{1}, X_{2}, \ldots, X_{M} \mid \boldsymbol{\theta}^{(k)}\right)$ is called the likelihood function; it represents the relative joint conditional probability of observing the measured values $X_{1}, X_{2}, \ldots, X_{M}$ under the condition that the parameter vector coincides with the $k$ th sample $\theta^{(k)}=\left(\theta_{i}^{(k)}, \ldots, \theta_{N}^{(k)}\right)$. The prior probability of each sample is $P^{\prime}\left(\theta^{(k)}\right)=P^{\prime}\left(X_{m}^{\prime(k)}\right)=P^{\prime}\left(Y_{i}^{\prime(k)}\right)=$ constant, and $c_{1}$ is determined from the normalizing condition $\Sigma_{k} P^{\prime \prime}\left(Y_{i}^{(k)}\right)=1$.

The most difficult task is the determination of the likelihood function, representing the relative joint probability of observing the measured values $X_{1}, \ldots, X_{M}$. Although the latin hypercube sampling has been extended to problems in which the random parameters for creep are correlated (Bažant and $\mathrm{Xi}$ 1988), the analysis becomes considerably more complicated. We will assume for the sake of simplicity that the values $X_{1}, X_{2}, \ldots, X_{M}$ are statistically independent; i.e., uncorrelated. The longer the time interval, the weaker the correlation of creep or shrinkage values at the beginning and the end of the interval, and so the assumption of statistical independence should be more realistic if the measurements used for updating are spaced sparsely in time.

According to our assumption of statistical independence

$L\left(\mathbf{X} \mid \boldsymbol{\theta}^{(k)}\right)=L\left(X_{1}, X_{2}, \ldots, X_{M} \mid \boldsymbol{\theta}^{(k)}\right) \approx \prod_{m=1}^{M} f_{m}^{X}\left(X_{m} \mid \boldsymbol{\theta}^{(k)}\right)$

Substituting now this into Eq. 4 , and noting that $P^{\prime}\left(\theta^{(k)}\right)=$ constant, we obtain the result

$P^{\prime \prime}\left(Y_{i}^{(k)}\right)=P^{\prime \prime}\left(\boldsymbol{\theta}^{(k)}\right)=c_{0} p_{k}, \quad p_{k}=\prod_{m=1}^{M} f_{m}^{X}\left(X_{m} \mid \boldsymbol{\theta}^{(k)}\right) \ldots \ldots \ldots \ldots \ldots \ldots \ldots$

in which $c_{0}$ is a constant. This constant may be determined from the normalizing condition $c_{0}=\left(\Sigma_{k} p_{k}\right)^{-1} ; f_{m}^{X}\left(X_{m} \mid \boldsymbol{\theta}^{(k)}\right)$ represents the density distribution of the conditional probability to obtain any value $X_{m}$ under the condition that the random parameter values are $\theta_{1}^{(k)}, \ldots, \theta_{N}^{(k)}$. Note that this probability density is not the prior probability of $X_{m}$ and cannot be taken the same as $f_{m}^{X^{\prime}}\left(X_{m}^{\prime(k)}\right)$ for the prior, which characterizes the statistical scatter of the properties of all kinds of concretes in general. Rather, $f_{m}^{X}\left(X_{m} \mid \boldsymbol{\theta}^{(k)}\right)$ should be interpreted simply as a characteristic of the statistical scatter of the properties of one particular concrete. Generally, the standard deviation $s_{m}^{X}$ of $f_{m}^{X}\left(X_{m} \mid \boldsymbol{\theta}^{(k)}\right)$ will be smaller than that of the prior, $f_{m}^{X^{\prime}}\left(X_{m}^{\prime(k)}\right)$. The fact that the probability described by $f_{m}^{X}$ is conditional to $\theta^{(k)}$ means that it refers to one particular concrete, for which the uncertainty of $\theta_{m}^{(k)}$ is much reduced.

The foregoing relations hold in general for any probability distributions. To obtain the prior probability density distribution $f_{m}^{X^{\prime}}\left(X_{m}^{\prime(k)}\right)$, one may use many samples $\theta^{(k)}$ to generate many values $X_{m}^{\prime(k)}(k=1, \ldots, K)$, then construct from these values the density histogram and fit to it a suitably chosen formula. To obtain the posterior probability density distributions $f_{m}^{X^{\prime \prime}}\left(X_{m}^{\prime \prime}\right)$ and $f_{i}^{Y^{\prime \prime}}\left(Y_{i}^{\prime \prime}\right)$, one needs to construct the weighted density histograms of values $p_{k} X_{m}^{\prime \prime(k)}$ and $p_{k} Y_{i}^{\prime \prime(k)}$ calculated for all samples $\theta^{(k)}$ and then again fit to these histograms a suitably chosen formula. The posterior (updated) means and standard deviations of the predictions of $X_{m}^{\prime \prime}$ and $Y_{i}^{\prime \prime}$ are obtained as

$\bar{X}_{m}^{\prime \prime}=c_{0} \sum_{k} p_{k} X_{m}^{\prime(k)}, \quad s_{m}^{X^{\prime \prime}}=\left[c_{0} \sum_{k} p_{k}\left(X_{m}^{\prime(k)}-\bar{X}_{m}^{\prime \prime}\right)^{2}\right]^{1 / 2}$
$\bar{Y}_{i}^{\prime \prime}=c_{0} \sum_{k} p_{k} Y_{i}^{\prime(k)}, s_{i}^{Y^{\prime \prime}}=\left[c_{0} \sum_{k} p_{k}\left(Y_{i}^{\prime(k)}-\bar{Y}_{i}^{\prime \prime}\right)^{2}\right]^{1 / 2}$.

where $c_{0}=\left(\Sigma_{k} p_{k}\right)^{-1}$. If we assume all the distributions to be normal (Gaussian), which seems sufficient for practice, then the probability densities of the prior and of the likelihood are

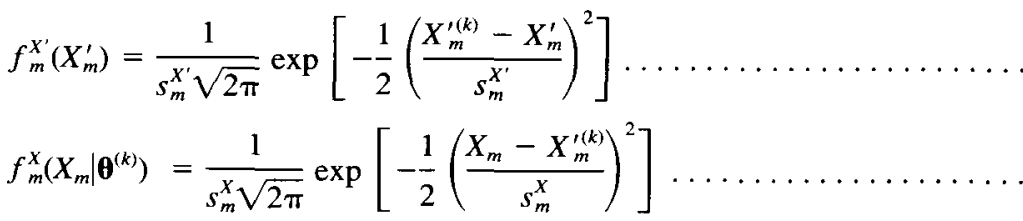

where $X_{m}^{\prime(k)}$ denotes the value calculated from the $k$ th parameter sample, $\boldsymbol{\theta}^{(k)}$. According to Eq. 6

$p_{k}=\exp \left[-\sum_{m=1}^{M} \frac{1}{2}\left(\frac{X_{m}-X_{m}^{\prime(k)}}{s_{m}^{X}}\right)^{2}\right]$

in which we dropped the multiplicative constant, since only the relative values of $p_{k}$ matter. In terms of $X_{m}^{\prime \prime}, s_{m}^{X^{\prime \prime}}, \bar{Y}_{i}^{\prime \prime}, s_{i}^{Y^{\prime \prime}}$ given by Eqs. $7 a-b$, the posterior normal probability density distributions are

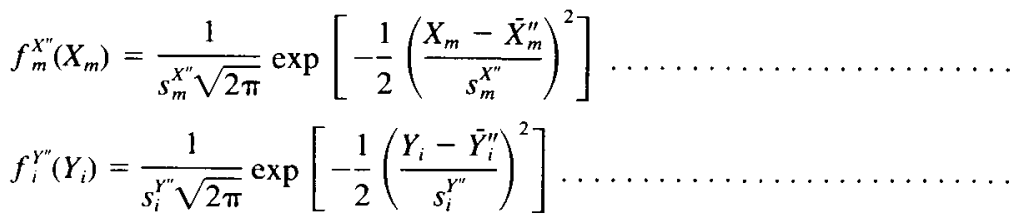

Typical cumulative histograms of the posterior response distribution, as obtained by the present sampling method, are shown in Figs. 5-6 in the normal probability scale. While the horizontal widths of all the steps of prior distribution in each of these histograms are the same, the step widths are unequal, since the weights of the samples are not the same. If the posterior probability distribution is normal, the histogram should be a straight line. Thus, the deviations from a straight regression line indicate how good the assumption of a normal probability distribution is. Figs. 5-6 confirm that this assumption is acceptable.

\section{Example of Typical Box-Girder Bridge}

As an example, we predict the long-time response of the Kishwaukee River Bridge in Illinois, introducing some simplifications. This is a segmental prestressed concrete box girder whose cross section and longitudinal section are shown in Fig. 1. The main data are summarized in Tables 2 and 3. The inner spans of the box girder have 19 segments per cantilever (i.e., per halfspan). The segments are placed symmetrically on both sides of the span. The cantilevers are joined at midspan at time $t_{m}=115$ days. The top tendons anchored in each segment are stressed at the moment of placement of that segment, while the continuity tendons are all stressed at the time of joining. The elastic modulus of the tendon is $E_{s}=27 \times 10^{6} \mathrm{psi}(186.2 \mathrm{GPa})$. The 
TABLE 2. Mean Values and Standard Deviations of Random Parameters Representing Prior Statistical Information on Creep and Shrinkage

\begin{tabular}{c|c|c|c}
\hline \hline $\begin{array}{c}\text { Random parameter } \\
(1)\end{array}$ & $\begin{array}{c}\text { Meaning } \\
(2)\end{array}$ & $\begin{array}{c}\text { Mean value } \\
(3)\end{array}$ & $\begin{array}{c}\text { Standard deviation } \\
(4)\end{array}$ \\
\hline$\theta_{1}$ & - & 1 & 0.14 \\
$\theta_{2}$ & - & 1 & 0.22 \\
$\theta_{3}$ & - & 1 & 0.13 \\
$\theta_{4}$ & $h$ & 0.65 & 0.05 \\
$\theta_{5}$ & - & 1 & 0.1 \\
$\theta_{6}$ & $w / c$ & 0.44 & 0.044 \\
$\theta_{7}$ & $g / c$ & 2.83 & 0.283 \\
$\theta_{8}$ & $c, \mathrm{~kg} / \mathrm{m}^{3}$ & 390 & 39 \\
\hline
\end{tabular}

girder carries its own weight plus $85 \mathrm{lb} /$ in. $(14.89 \mathrm{kN} / \mathrm{m})$ of additional dead load, which is assumed to be applied at the time $t_{r}$ when the bridge is opened to traffic ( $t_{r}=287$ days). A small portion of the design live load, equal to $10 \mathrm{lb} /$ in. $(1.75 \mathrm{kN} / \mathrm{m})$, is assumed to be applied permanently, contributing to creep.

For calculations, the cross section is subdivided into 18 layers [Fig. 1(d)]. The prestressing steel is treated as separate layers. The time steps for numerical integration are increased in a geometric progression with 5 steps per decade in log-scale. However, the first time step after placing each segment, after joining the cantilevers, and after applying the additional dead load, is always taken as 0.1 day.

TABLE 3. Data on Box Girder Analyzed (Kishwaukee River Bridge)

\begin{tabular}{c|c|c|c}
\hline \hline $\begin{array}{c}\text { Segment number } \\
(\mathbf{1})\end{array}$ & $\begin{array}{c}\text { Age (in days) at } \\
\text { time of placement } \\
(2)\end{array}$ & $\begin{array}{c}\text { Number of } \\
\text { top tendons } \\
(3)\end{array}$ & $\begin{array}{c}\text { Numbers of } \\
\text { bottom tendons } \\
(\mathbf{4})\end{array}$ \\
\hline 1 & 100 & 100 & 2 \\
2 & 101 & 95 & 2 \\
3 & 103 & 84 & 2 \\
4 & 103 & 76 & 2 \\
5 & 104 & 69 & 2 \\
6 & 104 & 60 & 2 \\
7 & 104 & 52 & 2 \\
8 & 105 & 44 & 8 \\
9 & 105 & 38 & 12 \\
10 & 105 & 26 & 18 \\
11 & 106 & 22 & 22 \\
12 & 106 & 19 & 26 \\
13 & 106 & 17 & 30 \\
14 & 106 & 15 & 34 \\
15 & 107 & 13 & 36 \\
16 & 107 & 11 & 36 \\
17 & 110 & 9 & 36 \\
18 & 110 & 7 & 38 \\
19 & 115 & & 40 \\
\hline \hline
\end{tabular}

For the sake of simplification, the deflections were calculated only for a single cantilever representing one-half of the inner span of the bridge. Therefore, the rotation of the cantilever at its support had to be estimated. This was done from deflections $\Delta_{1}$ and $\Delta_{2}$ measured on the site at certain points of the end span and of interior span, as shown in Fig. 1(e). Based on elastic calculation of the deflection curve, the ratio $r=\left(\Delta_{1}-\Delta_{2}\right)_{1} / L_{1} \theta$ has been calculated; $L_{1}=$ distance between the points of deflection $\Delta_{1}$ and $\Delta_{2}$ [Fig. $1(e)]$. The rotations at support in presence of creep were then estimated for various times as $\theta=\left(\Delta_{2}-\Delta_{1}\right) / L_{1} r$, where $\Delta_{1}$ and $\Delta_{2}$ are the deflections actually measured on the bridge. These rotation values were then considered as the support condition for creep analysis of the cantilever.

The statistical predictions have been calculated for sets of 8,16 , and 32 random samples of parameters $\theta_{i}$ (representing the number of computer runs). The results for Bayesian predictions with 8 and 16 samples in the set differed considerably, but the results with 16 and 32 were relatively close to each other (see Figs. 5-8). It was concluded that at least 16 random samples should be used in each set, which means that 16 deterministic creep analyses of the structure were run for each set.

To assess the reliability of the resulting statistics, the calculations were run for several different randomly generated sets of 16 or 32 parameter samples, and were then mutually compared. Figs. 5 and 6 show the cumulative distributions of the predicted deflection. The prior predictions are shown by the data points, all of which have equal weights. Due to unequal weights of the posterior predictions, the posterior predictions cannot be graphically shown as data points. Rather, the posterior cumulative distributions are shown in the form of cumulative histograms of a staircase shape (Figs. 5-6). The lengths of the horizontal steps on the staircase represent the weights of the predicted posterior values, and are therefore unequal, while for the prior prediction all the horizontal steps of the histogram would be equal (in the actual percentage scale, not in the normal probability scale used in figures).

The cumulative distributions of the deflection at the center of the 17 th segment at time $t=45$ years obtained for three different sets with 16 samples are plotted in Fig. 5, and then with 32 samples in Fig. 6. Figs. 5 $(a-c)$ and $6(a-c)$ show the histograms separately for each set of 16 samples, and Figs. $5(d)$ and $6(d)$ combine these histograms in one plot. Fig. $6(e)$ combines all the histograms with 16 and 32 samples. These figures show that the posterior predictions obtained are sufficiently close to each other for practical purposes. For 16 samples in each set, the differences among the results for various sets are $2.3 \%$ of the mean and $4.9 \%$ of the standard deviation for the prior distribution, and $1.3 \%$ of the mean and $17.7 \%$ of the standard deviation for the posterior distribution. For 32 samples, these differences are $3.4 \%$ and $21.4 \%$ for the prior distribution and $4.3 \%$ and $23.4 \%$ for the posterior distribution. According to these calculations, 16 random samples seem to suffice for practical purposes, although 32 random samples might be preferable. To improve the results, the final estimate of mean $\bar{Y}^{\prime \prime}$ and standard deviation $s^{\gamma^{\prime \prime}}$ are obtained as $\bar{Y}^{\prime \prime}=\sum_{\nu} \bar{Y}^{\prime \prime}{ }_{(\nu)} / N_{s}$ and $s^{Y^{\prime \prime}}=\left[\sum_{\nu} s_{(v)}^{Y^{\prime 2}} / N_{s}\right]^{1 / 2}$, where $v=1, \ldots, N_{s}$ labels the different sets of 16 or 32 samples.

The calculations described have been run for various times. This provided the prior and posterior predictions of response histories shown in Fig. 7 for the deflection of segment number 17 and the axial shortening displacement at the midspan after the opening of the bridge to traffic. The prior predictions 

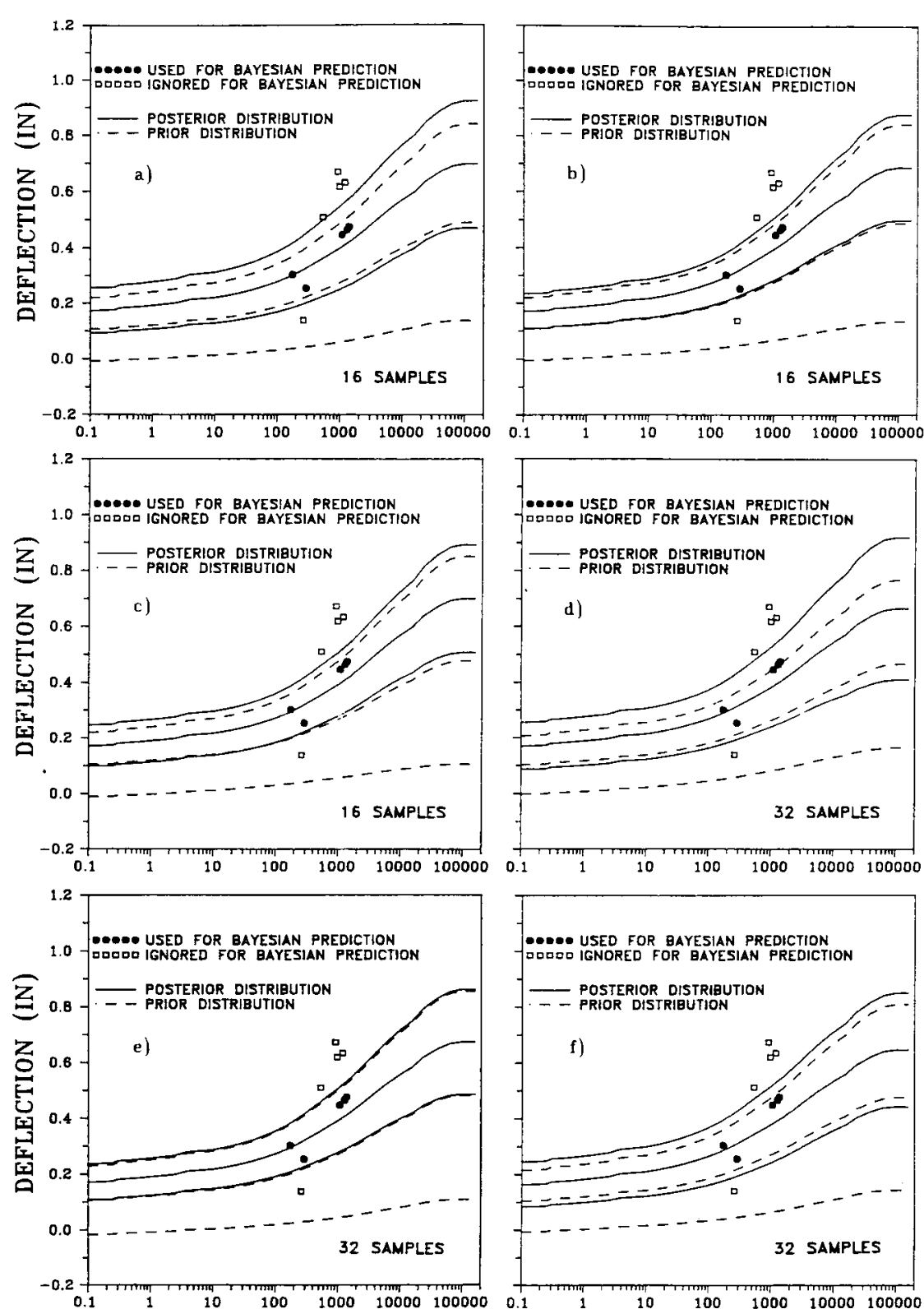

DAYS

FIG. 7. Prior and Posterior Predictions of Deflections of 17th Segment at $45 \mathrm{yr}$ : $(a-c)$ Based on 16 Samples in Each Set; $(d-f) 32$ Samples in Each Set
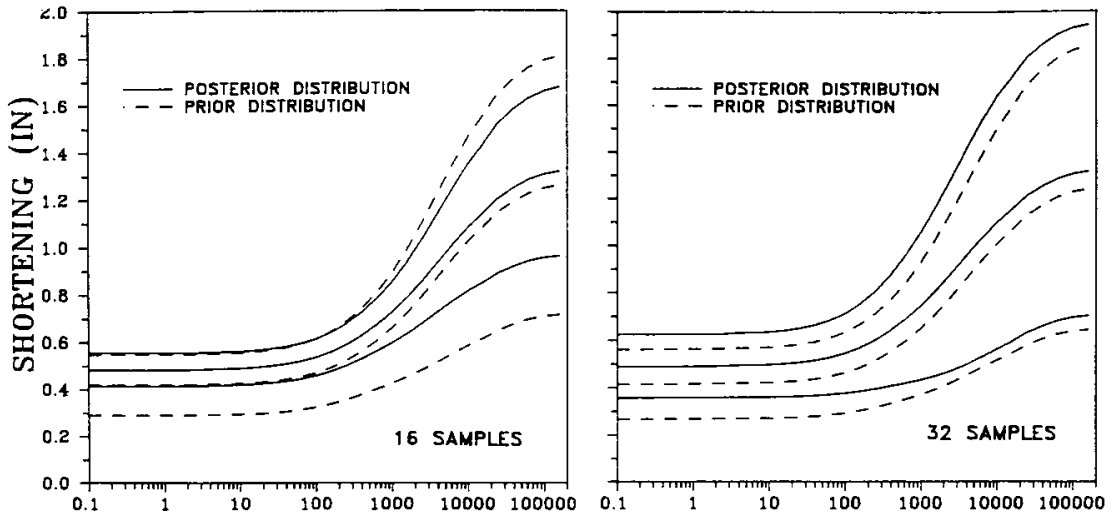

DAYS

\section{FIG. 8. Prior and Posterior Predictions of Span Shortening}

of long-time deflections and of shortening at the midspan are shown in Fig. 7 by their means and the bands of mean \pm two standard deviations, plotted as the dashed lines. These lines define a band of prior probability $95 \%$ (this means the prior probability of a value above the band is $2.5 \%$ and below the band also $2.5 \%$ ). The short-time data used for updating the prediction are shown in Fig. 7 as the data points. The updated, or posterior, predictions are again shown by their means and the bands of mean \pm two standard deviations, defining the $95 \%$ probability band, plotted as the solid lines. Fig. 8 shows the corresponding results for the axial shortening of the cantilever As might be expected, the width of the posterior probability band widens into the future, indicating an increase of prediction uncertainty with time.

The short-time deflections measured on the bridge are shown as the data points in Fig. 7. The measured deflections show very high scatter. Some of the measurements (the solid points) fall within the expected range, while others (the empty points) are far out of this range. Moreover, these out-ofrange points exhibit an unacceptable trend, since they violate the condition that creep can only increase. No doubt there were some further influences, such as temperature fluctuations and moisture content changes, that caused such behavior. Unfortunately, it is not possible to take such further influences into account, not only because of scope limitations of this study but also because measurements of the thermal and moisture conditions of the bridge are lacking. Therefore, the out-of-range measurements corresponding to the empty data points in Figs. 7 have been ignored in calculations; i.e., the Bayesian updating has been made only on the basis of the solid points.

The aforementioned problems show that a method to separate thermal and moisture effects from the measured short-time deflection data would have to be developed in order to achieve fully realistic results of updating that is based on measured short-time deflections of the bridge.

The bridge used as an example was selected because of the availability of documentation (Shiu et al. 1983), even though this bridge does not represent the case where the Bayesian updating of vertical alignment on the basis of deflection measurements is of the greatest value. This bridge was erected 
very fast, each half-span within about 15 days. Consequently, the measurements during erection give relatively little information on creep, and have a lesser importance for long-time deflection than measurements on a bridge erected slower, as is the case for some other methods of construction.

Segmental box-girder bridges should be designed not for the mean response, as is the practice now, but for extreme response, such as the $95 \%$ confidence limits (represented by the mean \pm two standard deviations). The present method makes it possible not only to make statistical predictions on the basis of the prior statistical information for all concretes from the literature, but also to update the predictions on the basis of deflection measurements made during construction. Based on such prediction, one should adjust during construction the vertical alignment of the remaining segments. However, to apply this method in practice, the effects of daily and seasonal fluctuations of environmental humidity and temperature must be eliminated from the short-time data used for updating, as already emphasized. This problem deserves further study.

As an alternative use of the present statistical approach, the prior predictions can also be updated on the basis of short-time laboratory measurements of concrete specimens of the particular concrete to be used in the structure. In that case, of course, the problem of separating the effect of random environmental fluctuations does not arise. This type of updating is certainly not as relevant as the measurements taken on the bridge during construction, but still is no doubt better than no updating at all, and its practical application is feasible right now.

It should also be recognized that our stress-strain relation in Eq. 1 is a simplified one. More realistically, the local values of pore humidity and temperature, which need to be solved from the diffusion equation, affect the creep rate. Nonuniformity of shrinkage and creep leads to residual stresses and cracking or tensile strain-softening. At present, it is not yet clear how important these effects are for the statistical predictions.

Finally, it should be kept in mind that the Bayesian approach itself is not perfect from the probabilistic viewpoint. The results depend only on the distributions of the prior and of the data but not on the number of prior data and the number of measurements used for the updating. Obviously, if the prior data consist of 10,000 points and the update consists of only 6 points, the prediction should be closer to the prior than if the update consists of 1,000 points, the prior data being same. But the Bayesian approach cannot distinguish between these two cases. In this respect, there is room for further fundamental improvement of the probability method.

\section{Alternative Method for Sharp or Remote Likelihood}

Our calculation procedure breaks down if the likelihood distribution is much sharper than the prior distribution or is too remote from the prior. In these situations, the response values for various parameter samples very seldom fall within the range of the observed data (the update), and an extremely large number of samples would be required to obtain a sufficient number (at least 8 ) of response values within the range of the data. In such a case, all coefficients $P_{k}$ are almost zero, and the results are meaningless.

An alternative procedure, also proposed by Bažant (1985), can cope with such situations, provided that the tails of the distributions can be assumed to be gaussian (normal). Paying, at first, no attention to the prior information, we need to extrapolate the given data $X_{m}$ to obtain predictions $Y_{i}$ for long times. This can be accomplished, e.g., by the Levenberg-Marquardt nonlinear optimization algorithm, for which a standard subroutine is available. Disregarding the prior information, this subroutine is used to calculate the optimum random parameter values $\theta_{n}=\bar{\theta}_{n}^{o}$, which minimize the sum of squares of the deviations of the response predictions $X_{m}^{o}$ from the observed data $X_{m}$ at times $t_{m}$. This subroutine not only estimates the optimum values of random parameters $\theta_{n}$, but it also estimates (on the basis of tangential linearizations) their standard deviations $s_{n}^{o}$. Then, assuming $\theta_{n}$ to have normal distributions with means $\bar{\theta}_{n}^{o}$ and standard deviations $s_{n}^{o}$, one can generate latin hypercube samples $\theta_{i}^{o(k)}$ of the random parameters $\theta_{n}$ on the basis of the updating data alone (i.e., without any regard to the prior). For each of these samples one can calculate the long-time responses $Y_{i}^{o(k)}(i=1,2, \ldots, I)$. Then, considering all the generated values $Y_{i}^{o(k)}$ for each time $t_{i}$, one can calculate their mean $\bar{Y}_{i}^{o}$ and standard deviation $s_{i}^{Y^{\circ}}$.

The extrapolated statistical information $Y_{i}^{o}$ and $s_{i}^{\gamma o}$ may be regarded as a set of update data for the long time $t_{i}$ and confronted in the Bayesian sense with the predictions $Y_{i}^{\prime}$ from the prior. For this purpose one needs to generate the prior latin hypercube samples $\theta_{n}^{\prime(k)}$ of random parameters $\theta_{n}$, and calculate the corresponding responses $Y_{i}^{\prime(k)}$ for all the samples. From these, one can then obtain the mean $Y_{i}^{\prime}$ and the standard deviation $s_{i}^{Y^{\prime}}$ of the prior prediction, for each time $t_{i}$, as described before.

Assuming normal distributions for both the extrapolated measured data and the predictions from the prior, we now have an elementary Bayesian problem, consisting of a combination of a normal prior with a normal likelihood (representing the extrapolated measurements). As is well known (Box and Tiao 1973:74), the posterior distribution is then also normal and its mean and standard deviation are given by

$Y_{i}^{\prime \prime}=\frac{\left(s_{i}^{Y^{\prime}}\right)^{-2} \bar{Y}_{i}^{o}+\left(s_{i}^{\gamma^{\prime}}\right)^{-2} \bar{Y}_{i}^{\prime}}{\left(s_{i}^{\gamma^{\prime \prime}}\right)^{-2}+\left(s_{i}^{\gamma^{\prime}}\right)^{-2}}$,

$\left(s_{i}^{\gamma^{\prime \prime}}\right)^{-2}=\left(s_{i}^{\gamma^{\prime \prime}}\right)^{-2}+\left(s_{i}^{\gamma^{\prime}}\right)^{-2} \ldots \ldots \ldots \ldots \ldots$

The measured short-time data $X_{m}$ are usually quite limited and do not suffice to obtain full statistical information on all the parameters $\theta_{n}$. Thus, if one attempts to determine from $X_{m}$ the optimum values $\bar{\theta}_{n}^{o}$ of all the parameters $\theta_{n}$, the problem is ill-conditioned, because very different values of $\theta_{n}$ yield nearly equally good fits of $X_{m}$, although the corresponding predictions $Y_{i}$ might be very different. In such a case, the number of random parameters $\theta_{n}$ for which the optimum values $\bar{\theta}_{n}^{o}$ are sought must be reduced, and for the remaining $\theta_{n}$ one must use the mean values of the prior. For rather limited update data $X_{m}$, one would optimize from $X_{m}$ only two parameters, $\theta_{1}$ and $\theta_{2}$ (and assume $\theta_{3}=\theta_{2}$ ). For more extensive update data $X_{m}$, one can optimize $\theta_{1}, \theta_{2}$, and $\theta_{3}$.

Keep in mind, too, that if the distribution of the data is extremely sharp, or if it is too remote from the prior, then Bayesian updating is of dubious value. A simple extrapolation of the given short-time data without any regard to the prior makes more sense.

\section{Conclusions}

1. Predictions of confidence limits for long-time deflections and internal forces can be improved by Bayesian analysis based on short-time measurements. 
2. The Bayesian analysis can in general be carried out by latin hypercube sampling of random parameters in the creep prediction model. No linearization with regard to the random parameters is necessary, and all the known random parameters of the creep prediction model, such as the BP model, can be considered.

3. The sampling approach reduces the probabilistic problem to a series of deterministic structural creep analyses for various samples of random material parameters. Differences in age between the bridge segments, as well as a change in the structural system from statically determinate to indeterminate, can be taken into account.

4. An example of Bayesian statistical predictions for one particular recently constructed bridge demonstrates feasibility of the proposed new method.

\section{ACKNOWLEDGMENT}

Partial financial support under U.S. National Science Foundation Grants No. FED-7400 and MSM-8815166 to Northwestern University is gratefully acknowledged. Thanks are due to K. N. Shiu of Construction Technology Laboratories, Skokie, Ill., for providing information on the construction data and measurements on the Kishwaukee River Bridge.

\section{Appendix. References}

ACI Committee 109/II. (1982). "Prediction of creep, shrinkage, and temperature effects in concrete structures." ACI SP-27, Designing for Effects of Creep, Shrinkage, and Temperature, ACI, Detroit, Mich., 51-93.

Ang, A. H.-S., and Tang, W. H. (1975). Probability concepts in engineering planning and design, vol. 1: Basic principles. John Wiley and Sons, New York, N.Y., 329-359

Bažant, Z. P. (1982a). "Mathematical models for creep and shrinkage of concrete." Creep and Shrinkage of Concrete Structures, Z. P. Bažant and F. H. Wittman, eds., John Wiley and Sons, London, U.K., 163-256.

Bažant, Z. P. (1982b). "Input of creep and shrinkage characteristics for a structural analysis program." Materials and Structures (Matériaux et Constructions), RILEM, 15(88), Paris, France, 283-290.

Bažant, Z. P. (1983). "Probabilistic problems in prediction of creep and shrinkage effects in structures." Proc., 4th Int. Conf. on Appl. of Statistics and Probability in Soil and Struct. Engrg. Florence, Italy, 325-356.

Bažant, Z. P. (1985). "Probabilistic analysis of creep effects in concrete structures." Proc., ICOSSAR 85, 4th Int. Conf. on Struct. Safety and Reliability. I. Konishi, A. H.-S. Ang, and M. Shinozuka, eds., Kobe, Japan, 1, 331-344.

Bažant, Z. P., et al. (1988). "Material models for structural creep analysis." Mathematical modeling of creep and shrinkage of concrete. Proc., RILEM Int. Symp. Northwestern Univ., Z. P. Bažant, ed., John Wiley and Sons, Chichester and New York, 99-216.

Bažant, Z. P., and Chern, J. C. (1984). "Bayesian statistical prediction of concrete creep and shrinkage." $A C I J ., 81(6), 319-330$.

Bažant, Z. P., and Liu, K. L. (1985). "Random creep and shrinkage in structures: Sampling." J. Struct. Engrg., ASCE, 111(5), 1113-1134.

Bažant, Z. P., and Panula, L. (1978a). "Practical prediction of time-dependent deformations of concrete," Materials and Struct. Parts I and II: 11(65), 307-328, Parts III and IV: 11(66), 415-434, Parts V and VI (1979): 12(69), 169-183.

Bažant, Z. P., and Panula, L. (1980). "Creep and shrinkage characterization for analyzing prestressed concrete structures." Prestressed Concrete Institute J., 25(3), 86-122.
Bažant, Z. P., and Panula, L. (1982). "New model for practical prediction of creep and shrinkage." ACI Special Pub., SP-76, Detroit, Mich., 7-23.

Bažant, Z. P., and Prasannan, S. (1988). "Solidification theory for aging creep." Cement and Concr. Res., 18(6), 923-932.

Bažant, Z. P., and Xi, Y. (1988). "Sampling analysis of concrete structures for creep and shrinkage with correlated random material parameters." Probabilistic Engrg. Mech., (in press)

Bažant, Z. P., and Wang, T. S. (1983). "Spectral analysis of random shrinkage stresses in concrete." J. Engrg. Mech., ASCE, 110(2), 173-186.

Bažant, Z. P., Kim, J.-K., Wittmann, F. H., and Alou, F. (1987). "Statistical extrapolation of shrinkage data-Part II: Bayesian updating." ACI Materials J., 84(2)

Bažant, Z. P., and Zebich, S. (1983). "Statistical linear regression analysis of prediction models for concrete creep and shrinkage." Cement and Concrete Research, 13(6), 869-876.

Benjamin, J. R., and Cornell, A. C. (1970). Probability, statistics and decision for civil engineers. Mc-Graw-Hill Book Co., New York, N.Y., 524-641.

Box, G. E. P., and Tiao, G. C. (1973). Bayesian inference in statistical analysis. Addison-Wesley Publishing Co., Reading, U.K.

CEB-FIP. (1978). Model code for concrete structures. Commité Eurointernational du Béton-Fédération Internationale de la Précontrainte, CEB Bulletin No. 124/ 125-E, Paris, France.

Ha, H., Osman, M. A., and Huterer, J. (1984). "User's guide: Program for predicting shrinkage and creep in concrete structures." Report. File: NK38-26191P, Ontario Hydro, Toronto, Canada.

Křístek, V., and Bažant, Z. P. (1987). "Shear lag effect and uncertainty in concrete box girder creep." J. Struct. Engrg., ASCE, 113(3), 557-574.

Madsen, H. O., and Bažant, Z. P. (1983). "Uncertainty analysis of creep and shrinkage effects in concrete structures." ACI J., 80(2), 116-127.

McKay, M. D. (1980). "A method of analysis for computer codes." Report, Los Alamos Nat. Lab., Los Alamos, N.M

McKay, M. D., Beckman, R. J., and Conover, W. J. (1979). "A comparison of three methods for selecting values of input variables in the analysis of output from a computer code." Technometrics, 21(2), 239-245.

McKay, M. D., Conover, W. J., and Whiteman, D. C. (1976). "Report on the application of statistical techniques to the analysis of computer code" Los Alamos Sci. Lab. Report LA-NUREG-6526-MS NRC-4, Los Alamos, N.M.

Shiu, K. N., Daniel, J. P., and Russell, H. G. (1983). "Time-dependent behavior of segmental cantilever concrete bridges." Report, Constr. Tech. Labs., Portland Cement Assoc., Skokie, IIl

Tsubaki, T., et al. (1988). "Probabilistic models." Mathematical modeling of creep and shrinkage of concrete. Proc RILEM Intern. Symp. Northwestern Univ $Z$ P. Bažant, ed., John Wiley and Sons, Chichester, and New York, 311-384. 